\title{
Intravesical Therapy for Urothelial Carcinoma of the Urinary Bladder: A Critical Review
}

\author{
Daher C. Chade, Shahrokh F. Shariat, Guido Dalbagni
}

Sloan-Kettering Institute (DCC) and Urology Service (SFS, GD), Memorial Sloan-Kettering Cancer Center, New York, NY, USA

\begin{abstract}
The management of non-muscle-invasive urothelial carcinoma of the bladder (UCB) is a challenge for physicians and patients alike. This is largely due to the heterogeneous natural history of this disease, in which tumors range from indolent to rapidly progressive and eventually fatal. Moreover, the high rate of recurrence and progression cause significant morbidity, expense, and detriment to quality of life. The advent of effective and safe intravesical therapies has improved the management of non-muscle-invasive UCB. Nevertheless, despite over 30 years of research and clinical experience, the mechanism, risks, benefits, and optimal regimens and treatment algorithms remain unclear. Although immunotherapy with bacillus Calmette-Guerin (BCG) has been the mainstay of intravesical treatment and represents a significant advance in the interaction of immunology and oncology, its clinical effectiveness is accompanied by a wide range of adverse events. Here, we review the literature on intravesical immunotherapy and chemotherapy with the aim of evaluating the clinical utility of the different treatments and providing recommendations. Many studies over the years have compared efficacy and toxicities of different agents and regimens, and certain conclusions are now well supported by high-level evidence. Future perspectives and promising advances in drug development are discussed and areas of improvement are identified in order to promote better cancer control and decrease the rate and severity of side-effects.
\end{abstract}

Key words: urinary bladder neoplasms; mycobacterium bovis; administration, intravesical; neoplasm recurrence, local; antineoplastic agents

Int Braz J Urol. 2009; 35: 640-51

\section{INTRODUCTION}

More than 357,000 new cases are diagnosed worldwide, and more than 145,000 deaths are related to urothelial bladder cancer (UBC) each year (1). The main risk factor for UBC is tobacco, which is thought to be responsible for at least one third of the cases. Males are three to four times more likely to develop UBC than their female counterparts. This discrepancy has been partially attributed to the higher proportion of smokers among males (1). At presentation, approximately $30 \%$ of patients have muscle-invasive UBC
(cT2 or higher) and $70 \%$ have non-muscle-invasive UBC, of which $70 \%$ is pTa disease, $20 \%$ is pT 1 disease, and $10 \%$ is carcinoma in situ (CIS) (2). Both the natural history of non-muscle-invasive UBC and its treatment strategies are highly variable. Although some patients never experience disease recurrence, others experience disease progression and eventually die of their disease (3). In the absence of intravesical treatment, a patient with non-muscle-invasive UBC has a $47 \%$ probability of disease recurrence within 5 years of diagnosis, and a $9 \%$ probability of progression to muscle-invasive disease within that period (4). 
There are few evidence and risk-based tools to help with decision-making for patients with non-muscle-invasive UBC. Factors predictive of outcome include clinical and pathologic features and molecular markers such as cytology, NMP22, and FISH. While management of cTa low-grade UBC is relatively non-controversial, the best management of patients with high-grade cTa, CIS, or cT1 UBC has not yet been established. The development and testing of effective intravesical therapies for non-muscle-invasive UBC are still evolving. Indeed, major controversies still exist with regard to the indication, type, and regimen of intravesical therapy. Other areas of controversy are the criteria for response/failure of treatment and for decisions regarding secondary intravesical therapy versus radical cystectomy. In this article, we analyze the different intravesical therapeutic strategies and critically compare their oncologic efficacy.

\section{Intravesical BCG Immunotherapy}

The first BCG strain was isolated in 1921 by Albert Calmette and Camille Guerin at the Pasteur Institute in France by attenuating the Mycobacterium bovis bacillus, resulting in a live vaccine against tuberculosis (5). Subsequently, laboratory and clinical studies demonstrated an anti-tumor effect of BCG against several malignant cell lines (6-9). In 1976, Morales et al. reported the first successful clinical study, where they evaluated nine patients with recurrent $\mathrm{UBC}$ treated with intravesical BCG once a week for 6 weeks, achieving a complete response in seven patients $(78 \%)(10)$. In the following years, both the Southwest Oncology Group (SWOG) and Memorial Sloan-Kettering verified the efficacy of the Morales regimen against UBC in larger, well-designed trials (11-14). These studies have led to the broad acceptance and application of BCG intravesical therapy for muscle-invasive UBC. Currently, multiple substrains of BCG are in use for intravesical immunotherapy throughout the world; these include Pasteur, ArmandFrappier, TICE, RIVM, Glaxo, Tokyo, as well as others (15). Several trials have compared strains, with different dosages and regimens, showing comparable clinical results (16).
Several well-designed clinical trials have directly compared transurethral resection (TUR) alone with TUR followed by induction BCG. They have unanimously demonstrated a statistically significant reduction of approximately $32 \%$ in UBC recurrence rates, with the rates for BCG-treated patients ranging between $20 \%$ and $57 \%$ at median follow-ups of 2 to 7 years $(11,17-20)$. The median time from TUR to first recurrence was prolonged from 1-2 years with TUR alone to 2 to 4 years with TUR plus intravesical therapy. It has been shown that the use of BCG was associated with a relative risk for UBC recurrence of $0.39(21,22)$. These encouraging results were sustained even in patients with recurrent or aggressive disease, including patients whose prior intravesical chemotherapy had failed $(11,23)$.

While reducing and/or delaying disease recurrence is an important endpoint for the management of patients with non-muscle-invasive UBC, an even more important endpoint is preventing progression to higher stage disease. Addition of intravesical BCG to TUR lowers the progression rate by a statistically and clinically significant margin. A large meta-analysis involving 4,863 patients from 24 clinical trials revealed a $27 \%$ reduction $(9.8 \%$ vs. $13.8 \%)$ in the odds of disease progression at a mean follow-up of 2.5 years for patients treated with TUR plus BCG (induction and maintenance) compared to those treated with TUR alone (24). The corresponding reduction in the risk of death due to bladder cancer was 19\%; this effect was, however, not statistically significant. More recently, a meta-analysis of 25 trials including 4,767 patients confirmed these results with an odds ratio of 0.61 for tumor recurrence with TUR plus BCG vs. TUR alone (25). Finally, combinations of BCG with other intravesical therapies have shown some early promise. The combination of BCG and interferon, for example, has shown some potential benefit, with recurrence-free rates of $59 \%$ and $45 \%$ in BCG-naïve and BCG-failure patients, respectively, within a 2-year median follow-up (26).

As with any treatment, optimal response depends on patient selection. Obviously, residual tumor after an incomplete TUR will result in treatment failure. Predictors of decreased response are stage cT1, multifocality, large tumor size, prior BCG failure, short time to previous BCG failure, and, most 
importantly, accurate staging using re-resection; however, none of these characteristics is an exclusion criterion. A repeat TUR within around 6 weeks of initial TUR will improve selection by re-classifying approximately $30 \%$ of understaged patients and eliminating the residual tumor in another 50\% (27).

With few exceptions, most investigators believe that the efficacy of BCG therapy can be maximized with maintenance therapy (28). Indeed, in an EORTC meta-analysis, Sylvester et al. reported that only trials involving maintenance therapy showed a significant decrease in disease progression for BCG plus TUR compared to TUR alone (odds ratio 0.63) (24). Even in patients with CIS alone, maintenance therapy with BCG results in the highest reduction of disease recurrence and progression rates (29). Therefore, based on these and other studies, the European Association of Urology and American Urological Association uniformly recommend at least a year of maintenance therapy for all high-risk patients getting BCG. The optimal maintenance schedule remains undecided. The SWOG program is the most widely applied schedule, with 3-week mini-series given at scheduled intervals of $3,6,12,18,24,30$, and 36 months (30).

The toxicities of BCG therapy vary from local urinary symptoms to severe inflammatory response. Most patients develop self-limited cystitis that may increase in intensity with later treatments $(31,32)$. Systemic manifestations present as fevers, chills, flu-like malaise, and rarely muscle and/or joint pain. Fever after BCG therapy is not always a sign of systemic BCG infection since most fevers are limited to 24 hours' duration (33). However, patients with fever lasting beyond 24 hours, especially if the fever persists more than 48 hours or has an intermittent evening pattern, are more likely to have systemic BCG infection. These patients usually require hospitalization and the administration of anti-tuberculosis agents, which in some cases should be accompanied by a short period of fluoroquinolone and systemic steroids (34). A progressively increasing symptomatology with each BCG cycle should prompt a delay, a lower dose, or interruption of BCG instillations, which may preclude long-term complications related to the immunotherapy. Reiter's syndrome (urethritis, arthritis, conjunctivitis) may occur during BCG treatment; if it does, interruption of the schedule is mandatory (35).

The tolerability of BCG can be improved by dose reduction, with one-third the standard dosage associated with a $30 \%$ to $50 \%$ reduction in toxicity with near equivalent efficacy (36).

\section{Intravesical Chemotherapy}

Several antineoplastic agents have been tested for the treatment of non-muscle-invasive UBC. Mitomycin C (MMC) is the most commonly used intravesical chemotherapy to date. Alternative agents are gemcitabine, doxorubicin, and epirubicin (not approved for clinical use in North America).

MMC is an anti-tumor antibiotic, which acts by inhibiting DNA synthesis. A review of nine randomized trials $(n=1,774)$ revealed that only five were able to show a statistically significant benefit in using intravesical MMC after TUR compared to TUR alone. The average recurrence rate was $54 \%$ in the TUR alone group versus $38 \%$ in the TUR plus MMC group (37). Dysuria and frequency were the most common side-effects, occurring in $41 \%$ of the patients (38). Response rates have varied widely across studies, due in part to differences in MMC preparation and protocol. Recently, Gao et al. demonstrated that tumor uptake and consequently oncologic efficacy of intravesical MMC were proportional to the drug concentration (39). In an attempt to optimize MMC delivery, a multi-institutional phase III trial was carried-out randomizing patients to the standard regimen versus the optimized regimen (40 $\mathrm{mg}$ MMC in 20 $\mathrm{mL}$ of sterile water, manipulations to reduce urine production, and alkalinization of urine). The recurrence rate at 5 years was decreased from $75 \%$ for the standard regiment to $49 \%$ for the optimized regimen. Moreover, the median time to recurrence was delayed from 12 to 29 months (40).

The optimization of intravesical chemotherapy with MMC consists of increasing the urinary $\mathrm{pH}$, reducing the volume of urine production and buffering the intravesical content. This is achieved by restricting fluids for 8 hours before and during the treatment, oral sodium bicarbonate starting 12 hours prior to, until immediately before the instillation, and 
emptying the bladder with an urethral catheter before instillation (40).

While MMC has been shown to decrease the risk for disease recurrence (by $\sim 14 \%$ ), the more important question for the management of patients with non-muscle-invasive UBC is whether MMC reduces tumor progression and mortality. A metaanalysis of 22 prospective randomized trials including 3,899 patients did not show any decrease in the risk of tumor progression with addition of MMC to TUR compared to TUR alone (41). Similarly, addition of MMC to TUR did not improve survival in an analysis of four EORTC and two Medical Research Council randomized trials including 2,535 patients with Ta and T1 UBC (4). Huncharek et al. performed a meta-analysis of 11 randomized trials comparing patients treated with intravesical chemotherapy after TUR versus TUR alone; the study focused on primary TUR, excluding patients with recurrent disease (42). The authors reported that addition of chemotherapy to TUR decreased the risk of tumor recurrence at one year by $44 \%$. Patients receiving chemotherapy for two years showed the greatest decrease in recurrence rates. In a follow-up meta-analysis of eight chemotherapy studies focusing on patients with recurrent tumors, Huncharek et al. found a 38\% reduction in the risk of disease recurrence at one year; this rate improved with prolonged treatment beyond 2 years (43). In these studies, doxorubicin appeared to be less effective than MMC.

Although earlier reports suggested that the beneficial effects of adjuvant intravesical chemotherapy are temporary, several studies have since demonstrated durable effects. A trial comparing one and five instillations of MMC after TUR versus TUR alone demonstrated a decrease in the recurrence rate after a median follow-up of seven years $(20,42)$. Similarly, a phase III trial comparing a standard versus an optimized dose of MMC showed a decreased recurrence rate at five years for the optimized dose (12). The role of maintenance chemotherapy and sequential chemo-immunotherapy, however, remains unclear.

The common indications of adjuvant intravesical chemotherapy instillations are directly related to the risk of tumor recurrence and progression. Despite no clear evidence of reducing progression rates with chemotherapy, classifying the patients according to their risk is essential to improve the outcomes. Both intermediate- and high-risk groups, defined by multiple tumors, tumor size $>3 \mathrm{~cm}$, prior recurrence rate, T1, CIS, and grade (EORTC risk tables), are eligible for intravesical chemotherapy. However, patients at high-risk of progression should certainly consider intravesical immunotherapy, due to the lack of evidence supporting the efficacy of chemotherapy in this setting $(42,43)$.

Finally, although single instillations are not the focus of this review, ample evidence shows that the immediate single post-operative instillation of chemotherapy reduces the recurrence rate when compared to TUR alone. Authors have shown a recurrence risk reduction by half at 2 years of follow-up and over $15 \%$ reduction at 5 years, rendering a routine recommendation for single post-operative instillation of MMC in Ta low-risk patients (44). The timing of the instillation has previously been evaluated by a large meta-analysis of randomized clinical trials, which showed to be sufficient if performed within the first 24 hours after the TUR (45). Complications have been rarely reported, except when bladder perforation occurs (44). The results are best in patients with a single small tumor that was entirely resected (45). However, this has shown a relatively low acceptance by the urological community to the routine use in clinical practice.

Recently, Dalbagni et al. tested the efficacy of intravesical gemcitabine in patients with BCG-refractory, high-risk non-muscle-invasive bladder cancer in a phase II prospective trial. Results showed that 50\% of patients had a complete response and $21 \%$ were free of disease at one year (46).

\section{Comparison of Intravesical Chemotherapy and Immunotherapy}

We will focus on the comparison between BCG and MMC since several studies have shown the superiority of BCG to other chemotherapeutic agents (47). A multitude of studies has compared BCG to MMC. While some have found no significant differences between BCG and MMC, others have shown a greater reduction in recurrence and progression rates with BCG. This discrepancy may be attributable, in 
part, to differences in study design, patient selection, tumor biology, regimen, and dosages. We note that none of these studies used the optimal MMC regimen discussed above. Nevertheless, in patients with CIS, the verdict is largely in favor of BCG over MMC, regardless of whether the regimen included maintenance therapy.

BCG was superior to MMC in time to recurrence in two reported large meta-analyses. In a metaanalysis involving 2749 patients with intermediate- to high-risk tumors, Böhle et al. found a significant superiority of BCG over MMC, with $61 \%$ of the patients in the BCG group and 53\% in the MMC group being recurrence-free after a median follow-up of 29 months (48). The odds ratio was 0.56 in favor of BCG (48). Interestingly, the recurrence-free advantage of BCG was only seen in those studies that used BCG maintenance (odds ratio 0.43 for $B C G$ with maintenance vs. MMC). The trade-off, though, was a 1.8-fold increased risk of cystitis in patients treated with BCG (53.8\% vs. 39.2\%). Shelley et al. found no significant difference in the efficacy between BCG and MMC therapy in their overall meta-analysis of 1,901 patients (49); however, they reported a highly significant reduction in recurrence in favor of $\mathrm{BCG}$ in a subgroup analysis involving patients with highly recurrent disease. Böhle et al. concluded that at least 12 BCG instillations or one-year duration of therapy were needed to achieve the significant superiority of BCG over MMC.

With regard to disease progression, the results for BCG versus MMC are less clear. Sylvester et al. were able to demonstrate a statistically significant advantage of BCG versus MMC for disease progression $(\mathrm{OR}=0.73)(24)$. Also, Böhle et al., using a large database, found a statistically significant reduction in the odds ratio of disease progression for patients treated with $\mathrm{BCG}$ compared to those treated with maintenance $\mathrm{MMC}(\mathrm{OR}=0.66)(50)$.

Given the non-conclusive evidence, MMC should be considered a viable alternative for patients with papillary tumors at low or intermediate risk of disease progression. Nevertheless, we lack level I evidence for this assumption as there are no prospective clinical trials of optimized MMC.

The clinical management decision in UBC involves the assessment of individual risk for recur- rence and progression (3). In the low-risk patients, a single immediate instillation of chemotherapy is recommended (45). However, in the Ta low-grade group, other possible options are no adjuvant therapy or an induction course of chemotherapy, while for high-grade or T1 disease BCG is the preferred option. Either BCG or MMC is recommended for patients with an increased risk of recurrence but with a low risk of progression (51).

\section{Induction versus Maintenance Regimens for Immunotherapy}

Two early randomized studies compared no maintenance to maintenance with either one dose of BCG every 3 months or one dose monthly for 2 years. Neither trial demonstrated a statistical advantage to maintenance therapy $(52,53)$. Furthermore, patients in both trials had additional local toxicity attributable to BCG maintenance. Palou et al., in a large randomized Spanish trial, reported an $11 \%$ overall benefit of routine 6 -week courses every 6 months for 2 years in patients with no evidence of disease 6 months after TUR and induction BCG, but this difference did not reach statistical significance (54).

Despite the negative results of these early trials, the SWOG 8507 trial, which was specifically designed to answer the maintenance question, indicated the utility of BCG maintenance, although for an alternative schedule (30). Patients were randomized to no maintenance versus maintenance using mini-series of three weekly treatments administered at 3 and 6 months, then every 6 months for 3 years. Over a oneyear follow-up, there was a statistically significant difference in favor of maintenance therapy. Among the 233 patients with CIS, a complete response occurred in $84 \%$ with maintenance BCG therapy versus $68 \%$ of patients without $(\mathrm{P}=0.004)$. Among 254 patients with papillary disease and complete resection at the time of randomization, $87 \%$ of the patients in the maintenance arm were disease-free at two years compared to $57 \%$ without maintenance. A differential recurrence-free survival rate of at least $20 \%$ persisted up to 5 years. For patients with CIS or papillary disease, median recurrence-free survival was roughly doubled in the maintenance arm, from 36 to 77 months. Treatment 
with maintenance BCG in fact lowered the progression rate by a statistically significant margin of $6 \%$. However, one quarter of patients on maintenance therapy experienced grade 3 toxicity, and less than half completed more than three cycles, with only $16 \%$ completing all seven planned cycles. Since the maintenance group as a whole benefited even without most patients completing a full 3 years of therapy, maximum benefit may have been achieved earlier.

Additional, indirect support for BCG maintenance has come from large meta-analyses of prior clinical trials. As noted above, Sylvester et al. found that only trials employing maintenance therapy contributed to the observed benefit of BCG over MMC (24). Similarly, Böhle et al. found that a statistically significant improvement in tumor recurrence favoring BCG over MMC was apparent only in trials using at least one year of BCG maintenance (48).

Malmström et al. recently published a metaanalysis including 9 trials and 2820 patients (55). Compared to patients receiving $\mathrm{MMC}$, patients receiving $\mathrm{BCG}$ with maintenance had a $32 \%$ reduction in risk of recurrence, while patients receiving $\mathrm{BCG}$ without maintenance had a $28 \%$ risk increase. Progression did not differ significantly for either BCG treatment or MMC.

Collectively, these data suggest that BCG maintenance should be considered in patients with high-risk non-muscle-invasive UBC.

\section{Induction versus Maintenance Regimens for Chemotherapy}

In a prospective multicenter study, Huland et al. found no significant difference in recurrence rate among patients treated with and without maintenance MMC or doxorubicin (56). Two EORTC prospective randomized trials comparing early versus delayed and short-term versus long-term (6 versus 12 months) treatment with $\mathrm{MMC}$ and doxorubicin found no significant difference in the disease-free interval between any of these groups. However, the recurrence rate was worse among patients with delayed treatment and no maintenance (57). This was further confirmed by a randomized trial of maintenance versus no maintenance after early instillation of epirubicin, showing no difference in disease recurrence (58). In contrast, Koga et al. reported a higher efficacy for long-term instillation of epirubicin versus short-term instillation (59). In a prospective randomized trial, the patients received their first treatment within 24 hours of TUR, followed by epirubicin for 3 months or 12 months. The 3 -year recurrence rate was $36 \%$ in the 3 -month group versus $15 \%$ in the 12 -month group. Conrad et al. similarly found that 3 years of monthly MMC maintenance was superior to no maintenance (recurrence $14 \%$ vs. $31 \%$ ) in Ta G2/3 and T1 G1-3 tumors at median follow-up of 2.9 years (60). In a meta-analysis of 11 randomized trials, Huncharek et al. suggested that chemotherapy for 2 years had the greatest effect on decreasing the recurrence rates (42). Given these mixed results, the role of maintenance chemotherapy is not yet clear. Further prospective randomized trials are needed before recommendations can be made based on high-level evidence.

More recently, a multi-institutional randomized phase four trial compared short- and long-term prophylaxis with MMC versus short-term immunoprophylaxis with BCG in 495 patients (61). In intermediate and high-risk non-muscle-invasive UBC patients, long-term MMC significantly reduced the risk of tumor recurrence without increasing adverse events, with 3-year recurrence-free rates of $86.1 \%$ for long-term MMC and $65.5 \%$ and $68.6 \%$ for short-term BCG and MMC.

The guidelines and consensus panels on non-muscle-invasive UBC (American Urological Association, National Comprehensive Cancer Network, European Association of Urology) did not agree on the optimal maintenance schedule and duration and therefore do not make any recommendations. However, the best available data support the use of a six-week induction course of BCG followed by a maintenance course for at least one year, when compared to standard MMC treatment $(48,50)$. Nonetheless, there are no reported studies evaluating optimized MMC regimen in a maintenance schedule in this setting (51). Moreover, despite published data supporting the use of maintenance BCG for non-muscle-invasive UBC, the issue remains unclear, since other randomized trials analyzing induction alone found evidence of comparable benefits in reducing the progression rate (62). Furthermore, most of the cases of BCG intolerance 
occur during maintenance therapy, contributing to the reluctance by urologists to use this regimen (63).

Intravesical therapy failures indicate the need to include radical cystectomy as an option in the management decision, since only half of the patients will respond to conservative treatment when recurrence is detected at 3 months after a BCG course (64). In this setting, no strong evidence supports the use of chemotherapy as a first option, unless the patient has shown evident signs of BCG intolerance. Ultimately, the risk of BCG toxicity should always be considered when recommending immunotherapy, and individual assessment is crucial when selecting therapy for patients at higher risk for recurrence and progression.

\section{Future Perspectives}

A great need for improvements is still awaited for the treatment of patients with UBC. Apart from defining the best regimen with the available drugs, efforts to increase efficacy have included several promising attempts to introduce new agents to intravesical therapy, to combine them with established agents, or to modify current regimens. Future potential means to improve BCG efficacy can be envisioned based on mechanistic considerations. One attractive mechanism is enhancing the T helper type 1 (TH1) regulatory cytokine cascade. Toward this goal, the activity of inhibitory mediators such as interleukin10 and PGE2 can be decreased by interferon- $\gamma$ and nonsteroidal anti-inflammatory drugs, respectively. Addition of stimulatory cofactors such as interferon- $\gamma$, interleukin-2, and GMCSF have similarly been shown to increase the TH1-inducing effects of BCG (65). Identifying $\mathrm{BCG}$ resistance mechanisms remains a high priority. A novel possibility for boosting BCG anti-tumor activity is enhancing effector processes via death domain receptors/apoptosis signaling suspected of being operative during BCG therapy. While some drugs such as COX-2 inhibitors have been found to promote these effects, novel inhibitors of apoptosis inhibitors (such as survivin, XIAP) have the potential for doing even more. Finally, there is the prospect of genetically modifying BCG's properties to express tumor-associated antigens, thereby creating a more specific cancer vaccine.
Aiming to further increase the antineoplastic immune response, investigators have developed several recombinant vaccines. A recombinant BCG that expresses pertussis toxin, tested in an orthotopic animal model, had significant impacts on tumor weight and survival $(66,67)$. Also, data published by Liu et al. suggest promising results with recombinant BCG that secretes human interferon- $\alpha 2 \mathrm{~B}$, which stimulates TH1-type immune response (68).

Several novel compounds have been proposed for the management of non-muscle-invasive bladder cancer. An agent derived from the mycobacterial cell wall-DNA complex has entered clinical testing. Patients receiving 4 and $8 \mathrm{mg}$ achieved complete response rates of $27.3 \%$ and $46.4 \%$, respectively (69). Although recent studies have been reported for several other compounds, as yet no published evidence supports their clinical use.

\section{SUMMARY}

Maintenance intravesical BCG immunotherapy results in a sustained and significant long-term reduction of disease recurrence in intermediate- and high-risk UBC patients. Three meta-analyses confirmed that BCG after TUR is superior to TUR alone or to TUR and chemotherapy in preventing recurrences. Two meta-analyses demonstrated that maintenance BCG prevents, or at least delays, the risk of tumor progression, suggesting that progression-free survival seems to be improved. Clinical guidelines recommend that patients at intermediate or high risk of recurrence and at intermediate risk of progression should be treated with BCG or MMC. This recommendation is based on meta-analyses showing that chemotherapy delays the time to first recurrence after TUR. Chemotherapy has not, however, been shown to influence either the time to progression to muscle-invasive disease or survival. Despite currently perceived superiority of BCG therapy might be an artifact resulting from prior chemotherapy failures reported in several studies, most evidence has pointed to higher efficacy for BCG compared to most chemotherapies. A meta-analysis of seven trials concluded that tumor recurrence was significantly reduced with BCG compared to MMC only in the subgroup of patients 
at high risk of tumor recurrence. In addition, only BCG regimens that include maintenance appear to be superior to MMC, recently confirmed in another large meta-analysis. Nonetheless, no studies have shown statistically significant differences between BCG and $\mathrm{MMC}$ in progression, cancer-specific survival, or overall survival.

Choosing the best treatment for patients with non-muscle-invasive UBC depends on a number of factors. Patients with low-risk non-muscle-invasive tumors (small, single tumor of low grade) respond to intravesical therapy, but the low progression rate (less than $5 \%$ ) does not justify treatment. Intermediate-risk patients (recurrent and/or multifocal and/or large tumor of low grade) should undergo an initial trial of intravesical therapy with BCG or MMC. High-risk patients (CIS or cT1) should be treated with BCG, according to the guidelines of both the European and American Urological Associations $(51,70)$. To improve outcomes of patients with non-muscle-invasive UBC, better drugs, regimens, and molecular-based patient selection are still needed.

\section{ACKNOWLEDGEMENT}

Supported by The Sidney Kimmel Center for Prostate and Urologic Cancers. Chade is a post-doctoral research fellow in urologic oncology supported by CAPES. Shariat is a fellow in urologic oncology supported by NIH T32-CA82088.

\section{CONFLICT OF INTEREST}

None declared.

\section{REFERENCES}

1. Parkin DM: The global burden of urinary bladder cancer. Scand J Urol Nephrol Suppl. 2008; 218: 12-20.

2. Kirkali Z, Chan T, Manoharan M, Algaba F, Busch C, Cheng L, et al.: Bladder cancer: epidemiology, staging and grading, and diagnosis. Urology. 2005; 66: 4-34.

3. Sylvester RJ: Natural history, recurrence, and progression in superficial bladder cancer. ScientificWorldJournal. 2006; 6: 2617-25.
4. Pawinski A, Sylvester R, Kurth KH, Bouffioux C, van der Meijden A, Parmar MK, et al.: A combined analysis of European Organization for Research and Treatment of Cancer, and Medical Research Council randomized clinical trials for the prophylactic treatment of stage TaT1 bladder cancer. European Organization for Research and Treatment of Cancer Genitourinary Tract Cancer Cooperative Group and the Medical Research Council Working Party on Superficial Bladder Cancer. J Urol. 1996; 156: 1934-40, discussion 1940-1.

5. Crispen R: History of BCG and its substrains. Prog Clin Biol Res. 1989; 310: 35-50.

6. Mathé G, Amiel JL, Schwarzenberg L, Schneider M, Cattan A, Schlumberger JR, et al.: Active immunotherapy for acute lymphoblastic leukaemia. Lancet. 1969; 1: 697-9.

7. Morton D, Eilber FR, Malmgren RA, Wood WC: Immunological factors which influence response to immunotherapy in malignant melanoma. Surgery. 1970; 68: 158-63; discussion 163-4.

8. Zbar B, Bernstein ID, Bartlett GL, Hanna MG Jr, Rapp HJ: Immunotherapy of cancer: regression of intradermal tumors and prevention of growth of lymph node metastases after intralesional injection of living Mycobacterium bovis. J Natl Cancer Inst. 1972; 49: 119-30.

9. Coe JE, Feldman JD: Extracutaneous delayed hypersensitivity, particularly in the guinea-pig bladder. Immunology. 1966; 10: 127-36.

10. Morales A, Eidinger D, Bruce AW: Intracavitary Bacillus Calmette-Guerin in the treatment of superficial bladder tumors. J Urol. 1976; 116: 180-3.

11. Lamm DL: Bacillus Calmette-Guerin immunotherapy for bladder cancer. J Urol. 1985; 134: 40-7.

12. Mori K, Lamm DL, Crawford ED: A trial of bacillus Calmette-Guérin versus adriamycin in superficial bladder cancer: a South-West Oncology Group Study. Urol Int. 1986; 41: 254-9.

13. Herr HW, Pinsky CM, Whitmore WF Jr, Oettgen HF, Melamed MR: Effect of intravesical Bacillus Calmette-Guerin (BCG) on carcinoma in situ of the bladder. Cancer. 1983; 51: 1323-6.

14. Herr HW, Pinsky CM, Whitmore WF Jr, Sogani PG, Oettgen HF, Melamed MR: Experience with intravesical bacillus Calmette-Guèrin therapy of superficial bladder tumors. Urology. 1985; 25: 119-23.

15. Fonseca FP, Bachega W Jr, Zequi SC, Sarkis AS, Guimaraes G, Priante AV, et al.: Treatment of patients with superficial bladder cancer stratified by risk groups treated with lyophilized Moreau-Rio de Janeiro BCG 
strain. Int Braz J Urol. 2002; 28: 426-35; discussion 435-6.

16. Yoneyama T, Ohyama C, Imai A, Ishimura H, Hagisawa $\mathrm{S}$, Iwabuchi $\mathrm{I}$, et al.: Low-dose instillation therapy with bacille Calmette-Guérin Tokyo 172 strain after transurethral resection: historical cohort study. Urology. 2008; 71: 1161-5.

17. Melekos MD, Chionis H, Pantazakos A, Fokaefs E, Paranychianakis G, Dauaher H: Intravesical bacillus Calmette-Guerin immunoprophylaxis of superficial bladder cancer: results of a controlled prospective trial with modified treatment schedule. J Urol. 1993; 149: 744-8.

18. Krege S, Giani G, Meyer R, Otto T, Rübben H: A randomized multicenter trial of adjuvant therapy in superficial bladder cancer: transurethral resection only versus transurethral resection plus mitomycin $C$ versus transurethral resection plus bacillus Calmette-Guerin. Participating Clinics. J Urol. 1996; 156: 962-6.

19. Iantorno R, Nicolai M, Mastroprimiano G, Ballone E, Passamonti M, Cipollone G, et al.: Randomized prospective study comparing long-term intravesical instillation of BCG after transurethral resection and transurethral resection alone in patients with superficial bladder cancer. J Urol. 1999; 161(Suppl): 284.

20. Lamm DL: Long-term results of intravesical therapy for superficial bladder cancer. Urol Clin North Am. 1992; 19: 573-80.

21. Millán-Rodríguez F, Chéchile-Toniolo G, SalvadorBayarri J, Palou J, Vicente-Rodríguez J: Multivariate analysis of the prognostic factors of primary superficial bladder cancer. J Urol. 2000; 163: 73-8.

22. Shelley MD, Court JB, Kynaston H, Wilt TJ, Fish RG, Mason M: Intravesical Bacillus Calmette-Guerin in Ta and T1 Bladder Cancer. Cochrane Database Syst Rev. 2000: CD001986.

23. Witjes JA, Fransen MP, van der Meijden AP, Doesburg WH, Debruyne FM: Use of maintenance intravesical bacillus Calmette-Guérin (BCG), with or without intradermal $\mathrm{BCG}$, in patients with recurrent superficial bladder cancer. Long-term follow-up of a randomized phase 2 study. Urol Int. 1993; 51: 67-72.

24. Sylvester RJ, van der MEIJDEN AP, Lamm DL: Intravesical bacillus Calmette-Guerin reduces the risk of progression in patients with superficial bladder cancer: a meta-analysis of the published results of randomized clinical trials. J Urol. 2002; 168: 1964-70.

25. Han RF, Pan JG: Can intravesical bacillus CalmetteGuérin reduce recurrence in patients with superficial bladder cancer? A meta-analysis of randomized trials. Urology. 2006; 67: 1216-23.
26. Joudi FN, Smith BJ, O'Donnell MA; National BCGInterferon Phase 2 Investigator Group: Final results from a national multicenter phase II trial of combination bacillus Calmette-Guérin plus interferon alpha-2B for reducing recurrence of superficial bladder cancer. Urol Oncol. 2006; 24: 344-8.

27. Herr HW, Donat SM, Dalbagni G: Can restaging transurethral resection of $\mathrm{T} 1$ bladder cancer select patients for immediate cystectomy? J Urol. 2007; 177: 75-9; discussion 79 .

28. Herr HW: Is maintenance Bacillus Calmette-Guérin really necessary? Eur Urol. 2008; 54: 971-3.

29. Sylvester RJ, van der Meijden AP, Witjes JA, Kurth $\mathrm{K}$ : Bacillus calmette-guerin versus chemotherapy for the intravesical treatment of patients with carcinoma in situ of the bladder: a meta-analysis of the published results of randomized clinical trials. J Urol. 2005; 174: 86-91; discussion 91-2.

30. Lamm DL, Blumenstein BA, Crissman JD, Montie JE, Gottesman JE, Lowe BA, et al.: Maintenance bacillus Calmette-Guerin immunotherapy for recurrent TA, T1 and carcinoma in situ transitional cell carcinoma of the bladder: a randomized Southwest Oncology Group Study. J Urol. 2000; 163: 1124-9.

31. Orihuela E, Herr HW, Pinsky CM, Whitmore WF Jr: Toxicity of intravesical BCG and its management in patients with superficial bladder tumors. Cancer. 1987; 60: 326-33.

32. Berry DL, Blumenstein BA, Magyary DL, Lamm DL, Crawford ED: Local toxicity patterns associated with intravesical bacillus Calmette-Guérin: a Southwest Oncology Group Study. Int J Urol. 1996; 3: 98-100; discussion 101.

33. Lamm DL, Steg A, Boccon-Gibod L, Morales A, Hanna MG Jr, Pagano F, et al.: Complications of Bacillus Calmette-Guerin immunotherapy: review of 2602 patients and comparison of chemotherapy complications. Prog Clin Biol Res. 1989; 310: 335-55.

34. Steg A, Leleu C, Debré B, Boccon-Gibod L, Sicard $D$ : Systemic bacillus Calmette-Guerin infection in patients treated by intravesical BCG therapy for superficial bladder cancer. Prog Clin Biol Res. 1989; 310: 325-34.

35. Shoenfeld Y, Aron-Maor A, Tanai A, Ehrenfeld M: Bcg and autoimmunity: another two-edged sword. J Autoimmun. 2001; 16: 235-40.

36. Martínez-Piñeiro JA, Martínez-Piñeiro L, Solsona E, Rodríguez RH, Gómez JM, Martín MG, et al.: Has a 3 -fold decreased dose of bacillus Calmette-Guerin the same efficacy against recurrences and progression of T1G3 and Tis bladder tumors than the standard dose? 
Results of a prospective randomized trial. J Urol. 2005; 174: 1242-7.

37. Nilsson S, Ragnhammar P, Glimelius B, Nygren P; SBU-group. Swedish Council of Technology Assessment in Health Care: A systematic overview of chemotherapy effects in urothelial bladder cancer. Acta Oncol. 2001; 40: 371-90.

38. Thrasher JB, Crawford ED: Complications of intravesical chemotherapy. Urol Clin North Am. 1992; 19: 529-39.

39. Gao X, Au JL, Badalament RA, Wientjes MG: Bladder tissue uptake of mitomycin $\mathrm{C}$ during intravesical therapy is linear with drug concentration in urine. Clin Cancer Res. 1998; 4: 139-43.

40. Au JL, Badalament RA, Wientjes MG, Young DC, Warner JA, Venema PL, et al.: Methods to improve efficacy of intravesical mitomycin C: results of a randomized phase III trial. J Natl Cancer Inst. 2001; 93: 597-604.

41. Lamm DL, Riggs DR, Traynelis CL, Nseyo UO: Apparent failure of current intravesical chemotherapy prophylaxis to influence the long-term course of superficial transitional cell carcinoma of the bladder. J Urol. 1995; 153: 1444-50.

42. Huncharek M, Geschwind JF, Witherspoon B, McGarry R, Adcock D: Intravesical chemotherapy prophylaxis in primary superficial bladder cancer: a meta-analysis of 3703 patients from 11 randomized trials. J Clin Epidemiol. 2000; 53: 676-80.

43. Huncharek M, McGarry R, Kupelnick B: Impact of intravesical chemotherapy on recurrence rate of recurrent superficial transitional cell carcinoma of the bladder: results of a meta-analysis. Anticancer Res. 2001; 21: 765-9.

44. Witjes JA, Hendricksen K: Intravesical pharmacotherapy for non-muscle-invasive bladder cancer: a critical analysis of currently available drugs, treatment schedules, and long-term results. Eur Urol. 2008; 53: 45-52.

45. Sylvester RJ, Oosterlinck W, van der Meijden AP: A single immediate postoperative instillation of chemotherapy decreases the risk of recurrence in patients with stage Ta T1 bladder cancer: a meta-analysis of published results of randomized clinical trials. J Urol. 2004; 171: 2186-90.

46. Dalbagni G, Russo P, Bochner B, Ben-Porat L, Sheinfeld J, Sogani P, et al.: Phase II trial of intravesical gemcitabine in bacille Calmette-Guérin-refractory transitional cell carcinoma of the bladder. J Clin Oncol. 2006; 24: 2729-34.

47. Lamm DL, Blumenstein BA, Crawford ED, Montie JE, Scardino P, Grossman HB, et al.: A randomized trial of intravesical doxorubicin and immunotherapy with bacille Calmette-Guérin for transitional-cell carcinoma of the bladder. N Engl J Med. 1991; 325: 1205-9.

48. Böhle A, Jocham D, Bock PR: Intravesical bacillus Calmette-Guerin versus mitomycin $\mathrm{C}$ for superficial bladder cancer: a formal meta-analysis of comparative studies on recurrence and toxicity. J Urol. 2003; 169: 90-5.

49. Shelley MD, Wilt TJ, Court J, Coles B, Kynaston H, Mason MD: Intravesical bacillus Calmette-Guérin is superior to mitomycin $\mathrm{C}$ in reducing tumour recurrence in high-risk superficial bladder cancer: a meta-analysis of randomized trials. BJU Int. 2004; 93: 485-90.

50. Böhle A, Bock PR: Intravesical bacille CalmetteGuérin versus mitomycin $\mathrm{C}$ in superficial bladder cancer: formal meta-analysis of comparative studies on tumor progression. Urology. 2004; 63: 682-6; discussion 686-7.

51. Hall MC, Chang SS, Dalbagni G, Pruthi RS, Seigne JD, Skinner EC, et al.: Guideline for the management of nonmuscle invasive bladder cancer (stages Ta, T1, and Tis): 2007 update. Urol. 2007; 178: 2314-30.

52. Hudson MA, Ratliff TL, Gillen DP, Haaff EO, Dresner SM, Catalona WJ: Single course versus maintenance bacillus Calmette-Guerin therapy for superficial bladder tumors: a prospective, randomized trial. J Urol. 1987; 138: 295-8.

53. Badalament RA, Herr HW, Wong GY, Gnecco C, Pinsky CM, Whitmore WF Jr, et al.: A prospective randomized trial of maintenance versus nonmaintenance intravesical bacillus Calmette-Guérin therapy of superficial bladder cancer. J Clin Oncol. 1987; 5: 441-9.

54. Palou J, Laguna P, Millán-Rodríguez F, Hall RR, Salvador-Bayarri J, Vicente-Rodríguez J: Control group and maintenance treatment with bacillus Calmette-Guerin for carcinoma in situ and/or high grade bladder tumors. J Urol. 2001; 165: 1488-91.

55. Malmström PU, Sylvester RJ, Crawford DE, Friedrich M, Krege S, Rintala E, et al.: An individual patient data meta-analysis of the long-term outcome of randomised studies comparing intravesical mitomycin-C versus bacillus Calmette-Guérin for non-muscle-invasive bladder cancer. Eur Urol. 2009; 56: 257-8; discussion $\underline{258-9}$

56. Huland H, Klöppel G, Feddersen I, Otto U, Brachmann W, Hubmann H, et al.: Comparison of different schedules of cytostatic intravesical instillations in patients with superficial bladder carcinoma: final evaluation of a prospective multicenter study with 419 patients. J Urol. 1990; 144: 68-71; discussion 71-2. 
57. Bouffioux C, Kurth KH, Bono A, Oosterlinck W, Kruger CB, De Pauw M, et al.: Intravesical adjuvant chemotherapy for superficial transitional cell bladder carcinoma: results of 2 European Organization for Research and Treatment of Cancer randomized trials with mitomycin $\mathrm{C}$ and doxorubicin comparing early versus delayed instillations and short-term versus longterm treatment. European Organization for Research and Treatment of Cancer Genitourinary Group. J Urol. 1995; 153: 934-41.

58. Okamura K, Kinukawa T, Tsumura Y, Otani T, Itoh $\mathrm{H}$, Kobayashi $\mathrm{H}$, et al.: A randomized study of shortversus long-term intravesical epirubicin instillation for superficial bladder cancer. Nagoya University Urological Oncology Group. Eur Urol. 1998; 33: $285-$ 8; discussion 289.

59. Koga H, Kuroiwa K, Yamaguchi A, Osada Y, Tsuneyoshi M, Naito S: A randomized controlled trial of shortterm versus long-term prophylactic intravesical instillation chemotherapy for recurrence after transurethral resection of $\mathrm{Ta} / \mathrm{T} 1$ transitional cell carcinoma of the bladder. J Urol. 2004; 171: 153-7.

60. Conrad S, Friedrich MG, Schwaibold H, Pichlmeier U, Schildt K, Wolf N: Long term prophylaxis with mitomycin $\mathrm{C}$ (MMC) further reduces tumor recurrence compared to short term prophylaxis with MMC or bacillus Calmette-Guerin (BCG). J Urol. 2004; 171: [Abstract] 271.

61. Friedrich MG, Pichlmeier U, Schwaibold H, Conrad $\mathrm{S}$, Huland H: Long-term intravesical adjuvant chemotherapy further reduces recurrence rate compared with short-term intravesical chemotherapy and shortterm therapy with Bacillus Calmette-Guérin (BCG) in patients with non-muscle-invasive bladder carcinoma. Eur Urol. 2007; 52: 1123-29.

62. Cookson MS, Herr HW, Zhang ZF, Soloway S, Sogani PC, Fair WR: The treated natural history of high risk superficial bladder cancer: 15-year outcome. J Urol. 1997; 158: 62-7.

63. van der Meijden AP, Sylvester RJ, Oosterlinck W, Hoeltl W, Bono AV; EORTC Genito-Urinary Tract Cancer Group: Maintenance Bacillus CalmetteGuerin for Ta T1 bladder tumors is not associated with increased toxicity: results from a European Organisation for Research and Treatment of Cancer Genito-Urinary Group Phase III Trial. Eur Urol. 2003; 44: 429-34.

64. Herr HW, Dalbagni G: Defining bacillus CalmetteGuerin refractory superficial bladder tumors. J Urol. 2003; 169: 1706-8.
65. Luo Y, Chen X, O'Donnell MA: Role of Th1 and Th2 cytokines in BCG-induced IFN-gamma production: cytokine promotion and simulation of BCG effect. Cytokine. 2003; 21: 17-26.

66. Andrade PM, Chade DC, Borra RC, Nascimento IP, Villanova FE, Leite LC, et al.: The therapeutic potential of recombinant BCG expressing the antigen S1PT in the intravesical treatment of bladder cancer(). Urol Oncol. 2009; 7. [Epub ahead of print]

67. Chade DC, Borra RC, Nascimento IP, Villanova FE, Leite LC, Andrade E, et al.: Immunomodulatory effects of recombinant $\mathrm{BCG}$ expressing pertussis toxin on TNF-alpha and IL-10 in a bladder cancer model. J Exp Clin Cancer Res. 2008; 27: 78.

68. Liu W, O'Donnell MA, Chen X, Han R, Luo Y: Recombinant bacillus Calmette-Guérin (BCG) expressing interferon-alpha $2 \mathrm{~B}$ enhances human mononuclear cell cytotoxicity against bladder cancer cell lines in vitro. Cancer Immunol Immunother. 2009; 13. [Epub ahead of print]

69. Morales A, Phadke K, Steinhoff G: Intravesical mycobacterial cell wall-DNA complex in the treatment of carcinoma in situ of the bladder after standard intravesical therapy has failed. J Urol. 2009; 181: 1040-5.

70. Babjuk M, Oosterlinck W, Sylvester R, Kaasinen E, Böhle A, Palou-Redorta J, et al.: EAU guidelines on non-muscle-invasive urothelial carcinoma of the bladder. Eur Urol. 2008; 54: 303-14.

\section{Accepted after revision:}

June 20, 2009

\author{
Correspondence address: \\ Dr. Daher C. Chade \\ Sidney Kimmel Center for Prostate \& \\ Urologic Cancers \\ Memorial Sloan-Kettering Cancer Center \\ 353 East 68th Street \\ New York, NY 10065, USA \\ Fax: + 1646 422-4394 \\ E-mail: dchade@gmail.com
}




\section{EDITORIAL COMMENT}

In the review by Chade et al., the authors highlight our current state of knowledge with regards to intravesical therapy for urothelial carcinoma of the bladder. Since the landmark paper by Morales et al. [ref 10] in over 30 years pertaining to intravesical BCG in the management of bladder carcinoma, there have been significant advances in our understanding of the tumor biology and treatment indications for intravesical therapy. However, current instillation therapeutic agents have limitations including: 1) potentially delaying definitive therapy at a time when the disease may be curable, 2) their potential local and systemic toxicity, and 3) cost of repeated instillation therapy and subsequent requirement for disease surveillance. Certain patients with high-grade disease (clinical stage T1G3 with or without concomitant carcinoma in situ) refractory to repeated courses of induction BCG are best served with radical cystectomy. Similarly, certain histological variants of bladder carcinoma (e.g. micropapillary) are refractory to current intravesical agents and should be treated by early radical cystectomy. Although certain technical factors may optimize the efficacy of intravesical therapy (e.g. urine alkalinazation, dehydration, etc.), their treatment-specific outcomes have not significantly improved over the past decade. Hence, future clinical applications of intravesical therapy will likely need to take into account the clinical features and genetic signature of a bladder tumor in order to identify the patients best suited for intravesical therapy as well as select the most effective instillation agent and treatment regimen for that specific patient. This personalized approach to genitourinary oncology will likely revolutionize our practice patterns in the years to come.

Dr. Philippe E. Spiess Division of Urologic Oncology H. Lee Moffitt Cancer Center Tampa, Florida, USA E-mail:philippe.spiess@moffitt.org 